drome group B (ERCC6) ${ }^{9}$ and two of the seven complementation groups of xeroderma pigmentosum (XPB and XPD) ${ }^{10}$, encode helicases that take part in nucleotide excision repair.

The success of the human genome project has led to rapidly expanding resources for the gene mapping community, and of these the EST (expressed sequence tag) database is becoming increasingly important. The mapping of ESTs to their position in the genome to produce a transcript map will have enormous impact on the positional cloning of genes ${ }^{11}$. In the foreseeable future once a gene has been mapped to within 1-2 centimorgan (or megabases), details of the 50 or so cDNAs which map to that region will be available from a database providing both positional and functional information. All candidate genes could then be systematically screened for mutations. Although this 'factory' approach will be both scientifically profitable and hugely successful, there are those who mourn the passing of intelligent and innovative gene cloning, as demonstrated in the identification of $B L M$.

Richard Korn and Yashin Ramkissoon are in the Laboratory of Human Molecular Genetics, Department of Genetics, University of Cambridge, Downing Street, Cambridge CB2 3EH, UK.

\title{
A driver of glaciation cycles?
}

\section{Donald E. Brownlee}

As the Earth orbits the Sun, it collides with vast numbers of small particles amounting to over 10,000 tons of accreted extraterrestrial material a year. The bulk of this mass is composed of fragments of comets and asteroids, between 10 micrometres and 1 millimetre in diameter. This rain of primitive material from the outer Solar System onto the Earth may have played a role in the development of life $^{1}$, and evidence presented on page 600 of this issue suggests that it may also play a role in terrestrial glaciation cycles. Here, Farley and Patterson ${ }^{2}$ use ${ }^{3} \mathrm{He}$ abundances measured in a deep-sea sediment core to monitor variations in the accretion rate of interplanetary dust particles (IDP). What they have found are temporal changes that correlate with the $100-\mathrm{kyr}$ periodicity in the succession of ice ages on the Earth, one of the so-called 'Milankovitch' cycles.

To see why this finding will make climate scientists sit up, a little background on the glacial-interglacial cycles is needed. The basic idea developed by Milankovitch was that cyclical changes in the Earth's orbital eccentricity, in the tilt of its axis (obliquity) and in its precession, affect the distribution of solar radiation and thus draw in their wake changes to the growth and decay of ice sheets. At least some of the dominant frequencies seen in the geological record of ice ages do seem to be plausibly accounted for in this way, the periodicities at 19 and $23 \mathrm{kyr}$ being attributable to precession and the 41-kyr periodicity to obliquity changes. But in the past $800 \mathrm{kyr}$, the strongest signal in the climate record has been at a frequency of $100 \mathrm{kyr}$, and although this in principle corresponds with the eccentricity cycle, variations in eccentricity should produce the weakest of the orbital effects on incident solar radiation.

Earlier this year, Muller and MacDon- ald proposed ${ }^{3}$ that the answer to this puzzle might lie in a hitherto neglected parameter: the inclination of the Earth's orbital plane. Like the eccentricity, this should vary with roughly $100-k y r$ periodicity, but the changes would not be expected to affect the incident solar radiation directly. Instead, Muller and MacDonald suggested that the climate cycle might be affected through variations in the accretion rate of interplanetary dust. Farley and Patterson have now found evidence for such a variation.

Dust in the interplanetary medium is generated by asteroid collisions and the disintegration of comets. On timescales of $10^{4}$ to $10^{6}$ years, individual submillimetre particles are lost from the inner Solar System by collisional disruption and orbital decay induced by radiation pressure (the Poynting-Robertson effect). On average, the production and destruction mechanisms must balance, and one might expect that the particle flux reaching the Earth would be in a quasi-steady state with superimposed fluctuations due to particle generation episodes such as the appearance of large, active comets. Additional complications include the formation of a dust belt just outside the Earth's orbit ${ }^{4}$ and variations of the Earth's orbital parameters.

As suggested by Muller and MacDonald $^{3}$, changes in orbital inclination could modulate the flux of accreted dust with a $100-k y r$ period. But the new results measured by Farley and Patterson indicate that the accretion rate is lowest at times of highest inclination; in other words, the phase of variation is the opposite of what would be expected. The effect, if confirmed, cannot merely be a simple modulation caused by the inclination sweeping through a distribution of particles concentrated to the ecliptic.

IDPs contain substantial amounts of helium (up to concentrations equivalent to $1 \mathrm{~cm}^{3}$ per gram at STP). As was discovered over three decades ago ${ }^{5}$, the isotopic composition of this helium is very different from that of most terrestrial helium found in deep-sea sediments. The isotopic composition of the IDP $\mathrm{He}$ is similar to the primordial ratio whereas the terrestrial component is largely radiogenic and contains mainly ${ }^{4} \mathrm{He}$. The lighter isotope ${ }^{3} \mathrm{He}$ is an excellent tracer for extraterrestrial matter and is retained in sediments for long periods of time ${ }^{6}$, so Farley and Patterson use ${ }^{3} \mathrm{He}$ abundances throughout a sediment core to trace the accretion of extraterrestrial material.

A most interesting aspect of the data is that the implied flux is high and has a 100kyr variability only during the Quaternary period, which began around 2 million years ago. The flux was much lower for all of the preceding 70 million years. If correct, this indicates that the recent $100-\mathrm{kyr}$ $\mathrm{He}$ variability correlates with the recent development of the 100-kyr climate cycles. It is odd, however, that the highest $\mathrm{He}$ abundances are found at the top of the sediment column and it is possible that the results may be influenced by partial He loss in older sediments.

Overall, these data are interesting and important. If a true causal link can be established between the IDP flux and any climate cycle, then the real question will be, how this can happen? How can meteoritic material have an effect when it constitutes only a minuscule fraction of the total particulate mass loading of the atmosphere? It is unlikely that small particles that the $\mathrm{He}$ measurements actually detect in sediments could have much effect. By virtue of their small size and moderate entry velocity, these survive atmospheric entry intact. But highvelocity particles and those larger than 0.1 micrometre in diameter are partly or totally vaporized in the mesosphere, and the resulting vapours and fine condensates $^{7}$ may play a more effective role. Although the timescales are different, these findings are reminiscent of a mechanism suggested by Bowen many years ago for linking rainfall with meteor showers ${ }^{8}$. Farley and Patterson's results remind us once again that our planet's climate is at the mercy of its wider environment.

Donald E. Brownlee is in the Department of Astronomy, University of Washington, Seattle, Washington 91195, USA.

1. Anders, E. Nature 342, 255-257 (1989).

2. Farley, K. A. \& Patterson, D. B. Nature 378, 600-603 (1995).

3. Muller, R. A. \& MacDonald, G. J. Nature 377, 107-108 (1995).

4. Dermott, S. F. et al. Nature 369, 719-723 (1994)

5. Merrihue, C. Ann. N. Y. Acad. Sci. 119, 351-367 (1964).

6. Farley, K. A. Nature 376, 153-156 (1995).

7. Hunten, D. M., Turco, R. P. \& Toon, O. B. J. atmos. Sci. 37, 1342-1357 (1980).

8. Bowen, E. G. J. Meteorol. 13, 142-151 (1956). 